\title{
Quantitative analysis of optic nerve damage in idiopathic intracranial hypertension (IIH) at diagnosis
}

\author{
S. Bianchi Marzoli $\cdot$ P. Ciasca $\cdot$ M. Curone $\cdot$ \\ G. Cammarata - L. Melzi - A. Criscuoli • \\ G. Bussone • D. D’Amico
}

(C) Springer-Verlag Italia 2013

\begin{abstract}
Optic neuropathy secondary to idiopathic intracranial hypertension (IIH) may be a severe complication which must be early identified, adequately monitored and treated to avoid blindness. The aim of this study was to quantify optic nerve involvement at time of diagnosis in a prospectively series of IIH investigated at a single Institution and to identify objective parameters for early diagnosis and follow-up. 38 consecutive patients ( 9 men, 29 females, mean age 39.8 years) with IIH underwent a complete neuro-ophthalmological evaluation including standardized automated perimetry as functional measurement of optic neuropathy and spectral domain optical coherence tomography (SD-OCT) measurements to grade papilledema or optic nerve atrophy. An overall diagnosis of optic nerve involvement was made in 50 out of 76 eyes (66\%); ophthalmoscopic signs of papilledema were identified in 35 eyes (46\%) while optic disc pallor was found in $13(17 \%)$. In all patients mean visual field deviation (MD, $\mathrm{dB}$ ) was -7.2 (range 5.3-33.2). SD-OCT measurements of peripapillary retinal nerve fiber layer thickness (PRNFLT) and of macular ganglion cell complex thickness (MGCCT) obtained in 40 eyes (20 subjects) showed normal PRNFLT in 12 eyes (30\%), increased in 16 (40\%) and reduced in 12 eyes (30\%); normal MGCCT in 26 eyes
\end{abstract}

S. B. Marzoli $(\bowtie) \cdot$ P. Ciasca · G. Cammarata ·

L. Melzi · A. Criscuoli

Neuro-ophthalmology Service, Department of Ophthalmology, Istituto Auxologico Italiano IRCCS Foundation, Via Mercalli 30, 20122 Milano, Italy

e-mail: s.bianchimarzoli@auxologico.it

M. Curone · G. Bussone · D. D'Amico

Headache Unit, Department of Clinical Neuroscience, C. Besta

Neurological Institute and Foundation, Milano, Italy
$(65 \%)$, reduced in $14(35 \%)$. In all eyes average RNFLT was increased (mean $130 \mu \mathrm{m}$, range 219-59) and average MGCCT was decreased compared to normal values (mean $89.5 \mu \mathrm{m}$, range 198-65). Increased PRNFLT was associated with reduced MGCCT in 4 eyes (10\%) indicating early retrograde optic nerve damage. Decreased PRNFLT was associated with decreased MGCCT in 10 eyes ( $83 \%$ ). These results indicate that, in IIH patients, signs of optic neuropathy can be identified in more than half of cases, even without papilledema evidenced on ophthalmoscopic examination. Moreover, an SD-OCT analysis, which can be definitively useful to quantify optic nerve edema or atrophy, can show damage of retinal ganglion cells in an early phase of the disease.

Keywords Idiopathic intracranial hypertension - Optical coherence tomography . Visual fields · Papilledema . Optic atrophy

\section{Introduction}

Idiopathic intracranial hypertension (IIH) is a clinical syndrome of unknown etiology characterized by increased intracranial pressure without hydrocephalus or mass lesion [1]. IIH mainly affects young obese females, and in the majority of cases, it causes papilledema with headache, transient visual obscurations, diplopia and pulsatile tinnitus. The potential chronic nature of the disease, if not carefully monitored and treated, can lead to severe loss of visual function. Moderate-to-severe papilledema is fairly easy identified on ophthalmoscopy, while subtle papilledema requires an experienced clinician; moreover, up to $6 \%$ of IIH cases do not present with clinical papilledema although optic nerve damage can occur. 
Spectral domain optical coherence tomography (SDOCT) is a non-invasive imaging technique producing highresolution cross-sectional images of the retina that allows in vivo measurements of retinal thickness in the macular and around the optic nerve. SD-OCT imaging has become a valuable tool in identifying and monitoring peripapillary retinal nerve fiber layer thickness (RNFLT) in optic neuropathies [2]. Moreover, changes in macular thickness which relates for up to $40 \%$ to ganglion cells layer are an important parameter to quantitate atrophy in optic neuropathies.

SD-OCT has been suggested as a tool in monitoring the amount and evolution of PRNFLT in optic disc edema [2] in patients with IIH [3, 4]; however, no one investigated macular thickness as comparison parameter for optic nerve damage in IIH.

The aim of this study was use SD-OCT as a quantitative method to measure papilledema and optic neuropathy as compared to functional parameter of visual loss in newly diagnosed IIH.

\section{Subjects and methods}

38 consecutive patients with IIH diagnosed at the Neurological Institute C. Besta were enrolled in the study. Inclusion criteria were age $>18$ years, high CSF opening pressure measured by lumbar puncture, normal MRI and venography, normal CSF composition and normal neuroophthalmological examination except for papilledema or optic disc pallor. Exclusion criteria were competing medical, neurological or neuro-ophthalmological conditions. Patients underwent complete neuro-ophthalmological evaluation including standardized automated perimetry (SAP, Humphrey 30-2, Sita Standard) and spectral domain optical coherence tomography (SD-OCT RTVue100) analysis of PRNFLT and MGCCT. Clinical and instrumental data that were analyzed included: best-corrected visual acuity (BCVA, Snellen test), mean deviation (MD, $\mathrm{dB})$ at SAP, average PRNFLT and MGCCT $(\mu \mathrm{m})$ measured by SD-OCT and the presence of papilledema or atrophy at ophthalmoscopic examination.

\section{Results}

38 patients with IIH were recruited ( 9 men, 29 females, mean age 39.8 years).

At the diagnosis, the mean BCVA was 0.8 (range 1.0-0.01). Based on evidence of visual field defect, optic neuropathy was diagnosed in 50 eyes out of $76(66 \%)$; mean MD was $-7.2 \mathrm{~dB}$ (range 5.3-33.2). By ophthalmoscopy papilledema was found in 35 eyes (46\%), while optic disc pallor was present in $13(17 \%)$ and normal optic disc was described in 16 eyes (37\%).

20 subjects (40 eyes) underwent SD-OCT measurements. Mean average PRNFLT was greater than the upper normal statistical values given by the instrument software (130 $\mu \mathrm{m}$, range 219-59); while mean average MGCCT was lower than normal values $(89.5 \mu \mathrm{m}$, range 198-65).

PRNFLT was normal in 12 eyes ( $30 \%$ ), increased in 16 eyes $(40 \%)$ and reduced 12 eyes (30\%). MGCCT was normal in 26 eyes $(65 \%)$ and decreased in $14(35 \%)$.

Decreased PRNFLT was associated with decreased MGCCT in 10 eyes $(83 \%)$; increased PRNFLT was associated with reduced MGCCT in 4 eyes $(10 \%)$ indicating early retrograde optic nerve atrophy.

The data confirm the use of SD-OCT imaging as a noninvasive quantitative tool for diagnosing and monitoring papilledema in IIH [5, 6]. PRNFLT measurements by SDOCT can be more reliable to quantitate the amount of papilledema that ophthalmoscopy alone which is subjective and may reflect significant inter-observer variability.

Many studies showed a strong association between the amount of papilledema and severity of visual loss in patients with newly diagnosed IIH [7]. However, information provided by SD-OCT may diverge from analysis of visual function; compared to SAP, that is a sensitive test for visual loss, SD-OCT abnormalities can reveal early optic nerve damage also in cases without papilledema or visual field loss or when papilledema is not reversed.

Since MGCCT measurements indicated the presence of optic neuropathy independently to PRNFLT ones, the data support the use of SD-OCT analysis to quantitate retinal ganglion cells damage in an early phase of the disease.

In conclusion, our study suggests that SD-OCT imaging must be included as a non-invasive quantitative method of monitoring retinal layer thickening in papilledema, as it may enhance the diagnosis of subtle disc swelling. Of furthermore importance, with MGCCT measurements, SDOCT gives a more reliable estimate of early retrograde optic nerve atrophy, even in cases without papilledema or visual loss.

\section{References}

1. Skau M, Yri H, Sander B, Gerds TA, Milea D, Jensen R (2013) Diagnostic value of optical coherence tomography for intracranial pressure in idiopathic intracranial hypertension. Graefes Arch Clin Exp Ophthalmol 251:567-574

2. Skau M, Milea D, Sander B, Wegener M, Jensen R (2011) OCT for optic disc evaluation in idiopathic intracranial hypertension. Graefes Arch Clin Exp Ophthalmol 249:723-730

3. Rebolleda G, Munoz-Negrete FJ (2009) Follow-up of mild papilledema in idiopatic intracranial hypertension with optical coherence tomography. Vis Sci 50:5197-5200 
4. Karam EZ, Hedges TR (2005) Optical coherence tomography of the retinal nerve fibre layer in mold papilloedema and pseudopapilloedema. Br J Ophthalmol 89:294-298

5. Wall M, George D (1991) Idiopathic intracranial hypertension. A prospective study of 50 patients. Brain 114(Pt 1A):155-180

6. Rowe FJ, Sarkies NJ (1998) Assessment of visual function in idiopathic intracranial hypertension: a prospective study. Eye (Lond) 12(Pt 1):111-118
7. Digre KB, Nakamoto BK, Warner JE, Langeberg WJ, Baggaley SK, Katz BJ (2009) A comparison of idiopathic intracranial hypertension with and without papilledema. Headache 49:185-193 